\title{
Coupling an Ensemble of Electrons on Superfluid Helium to a Superconducting Circuit
}

\author{
Ge Yang, ${ }^{1}$ A. Fragner, ${ }^{2}$ G. Koolstra, ${ }^{1}$ L. Ocola, ${ }^{3}$ D. A. Czaplewski, ${ }^{3}$ R. J. Schoelkopf, ${ }^{2}$ and D. I. Schuster ${ }^{1, *}$ \\ ${ }^{1}$ The James Franck Institute and Department of Physics, University of Chicago, Chicago, Illinois 60637, USA \\ ${ }^{2}$ Department of Physics and Applied Physics, Yale University, New Haven, Connecticut 06520, USA \\ ${ }^{3}$ Argonne National Laboratory, Center for Nanoscale Materials, Argonne, Illinois 60439, USA
}

(Received 25 August 2015; published 21 March 2016)

\begin{abstract}
The quantized lateral motional states and the spin states of electrons trapped on the surface of superfluid helium have been proposed as basic building blocks of a scalable quantum computer. Circuit quantum electrodynamics allows strong dipole coupling between electrons and a high- $Q$ superconducting microwave resonator, enabling such sensitive detection and manipulation of electron degrees of freedom. Here, we present the first realization of a hybrid circuit in which a large number of electrons are trapped on the surface of superfluid helium inside a coplanar waveguide resonator. The high finesse of the resonator allows us to observe large dispersive shifts that are many times the linewidth and make fast and sensitive measurements on the collective vibrational modes of the electron ensemble, as well as the superfluid helium film underneath. Furthermore, a large ensemble coupling is observed in the dispersive regime during experiment, and it shows excellent agreement with our numeric model. The coupling strength of the ensemble to the cavity is found to be $\approx 1 \mathrm{MHz}$ per electron, indicating the feasibility of achieving single electron strong coupling.
\end{abstract}

DOI: 10.1103/PhysRevX.6.011031

\section{INTRODUCTION}

Electrons on helium are a promising resource for quantum optics and quantum computing [1-4]. They form an extremely clean two-dimensional electron gas [5], as evidenced by a mobility exceeding $10^{7} \mathrm{~cm}^{2} / \mathrm{V} \mathrm{s}[6,7]$, and the electron spin coherence time is predicted to exceed $10^{3} \mathrm{~s}$ [1]. Electrons on helium have been used to study Wigner crystallization and quantum melting [8-10]. Recent experiments employ them as a powerful probe to study the topological domain structures on the surface of superfluid helium 3 [11-13]. In addition, it is now possible to build mesoscopic structures such as quantum dots with one or a few electrons on helium, and single electron scale charge coupled devices [14-17]. However, performing quantum experiments in this fascinating system has lagged behind that in semiconducting 2D electron gases, such as GaAs, as traditional measurement techniques cannot be applied to electrons on helium. In particular, it is not possible to make direct Ohmic contact to the electron gas. Additionally, the largely unscreened electron-electron Coulomb force and a hydrostatic instability of the system [18,19] suppress the exchange interactions typically used in semiconductor spin qubits [20].

\section{*David.Schuster@uchicago.edu}

Published by the American Physical Society under the terms of the Creative Commons Attribution 3.0 License. Further distribution of this work must maintain attribution to the author(s) and the published article's title, journal citation, and DOI.
Subject Areas: Atomic and Molecular Physics,

Quantum Information,

Semiconductor Physics
The circuit QED architecture [21,22] offers a path to new experiments in the quantum regime as well as improving the sensitivity and bandwidth of existing measurements. In this hybrid approach, electrons are trapped above an on-chip superconducting microwave resonator. The presence of the electrons changes the effective capacitance of the cavity, resulting in a dispersive shift of the cavity resonance frequency. In the strong dispersive regime, the cavity frequency shift is larger than the cavity linewidth, and every photon measures the state of the electrons. Because the energy of a single photon in the cavity is higher than the thermal bath $\left(\hbar \omega>k_{b} T\right)$, it is possible to conduct quantum optics experiments at the single-photon level. This dispersive measurement is conceptually similar to the Sommer-Tanner technique [23], but the use of resonant superconducting circuits at microwave frequencies enables better impedance matching, resulting in faster and more sensitive measurements of small ensembles. Finally, the hybrid architecture allows one to leverage the substantial progress in superconducting circuits over the past decade [24,25].

While in this work we study the motional coupling of the electrons, they also carry a spin degree of freedom. Ensembles of electrons on helium could also be used as a quantum memory, similar to previous work on solid-state spin ensembles [26-29]. Uniquely, electrons on helium represent a mobile coherent spin system and can be dynamically arranged with gate electrodes. Finally, by engineering an artificial spin-orbit coupling it should be possible to achieve single-spin strong coupling [4,30-32]. 
In this paper, we report the first implementation of a circuit QED architecture with electrons on helium. We show lithographic control and subnanometer measurement of the superfluid helium film thickness. Our experiment shows a strong dispersive shift due to the electrons that is many times the cavity linewidth. On average, the coupling per electron in the ensemble is about $1 \mathrm{MHz}$, suggesting single electron strong coupling should be within reach. Electrons can be held for many hours, and their normal mode frequencies and number can be controlled by adjusting the trapping potential. The resulting evolution of the dispersive shift agrees excellently with our numerical model.

\section{EXPERIMENTAL SETUP AND DETECTION TECHNIQUE}

The electron on helium circuit QED setup consists of an integrated electron trap and coplanar waveguide resonator [see Fig. 1(a)]. The ground planes of the resonator are thicker than the center pin, forming a microcapillary channel that determines and stabilizes the superfluid helium film thickness [33,34]. The electrons are held in the resonator volume by a dc bias voltage applied to the center pin as shown in Fig. 1(c).

The electrons are confined in both the transverse and longitudinal directions of the channel. In the transverse direction, a dc voltage applied to the center pin creates a parabolic trapping potential [Fig. 1(c)] that confines the electron ensemble in the channel, colocated with the microwave field. In addition to the large electron trap formed by the resonator center pin, the devices also contain smaller $\mu \mathrm{m}$-size electron traps positioned near the voltage maxima of the fundamental mode for future single-electron experiments [Fig. 1(d)]. Those smaller traps are set to ground potential throughout the experiments discussed in the rest of this paper. The input and output of the resonator [Fig. 1(b)] side of the coupler are held at $0 \mathrm{~V}$ to prevent electrons from leaking out the sides. Along the cavity dc bias lead, where the potential may be positive, constrictions shield the electrons, ensuring that there is a potential barrier for escape.

The bare cavity resonance frequency is $\omega_{0} / 2 \pi \simeq$ $4.789 \mathrm{GHz}$, loaded quality factor $Q_{L} \simeq 17750$, and corresponding decay rate $\kappa / 2 \pi \simeq 270 \mathrm{kHz}$ in the absence of any superfluid or electrons. The $Q$ of the sample is set by the couplers, not by the internal $Q$ of the resonator, despite the fact that the dc bias lead directly connects the center pin to a low impedance. This is possible because the connection is made at a voltage node, where radiation is minimized [30,35].

\section{HELIUM DYNAMICS}

An important prerequisite for trapping electrons on helium in a microchannel geometry is to establish a selfstabilized film of superfluid helium of known thickness, which can be achieved by capillary action filling of the channels from the low-lying bulk reservoir. The helium
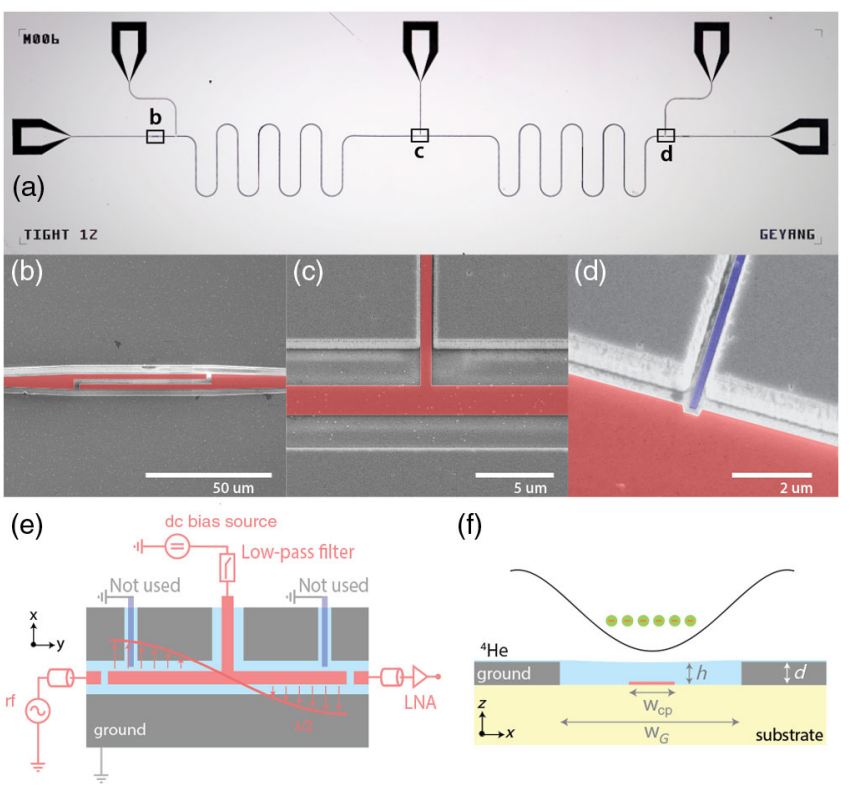

FIG. 1. Device, circuit schematic, and trap geometry. (a) Optical and SEM images of a cavity-electron ensemble trap on a $(2 \times 7)$ $\mathrm{mm}$ superconducting chip. The device is positioned $5.5 \mathrm{~mm}$ above the bottom of a cylindrical superfluid reservoir of radius $r=3.175 \mathrm{~mm}$, mounted in a hermetically sealed copper box at $25 \mathrm{mK}$ in a dilution refrigerator. (b) Interdigitated gap capacitors with gap width $2 \mu \mathrm{m}$ at the cavity input. (c) dc bias electrode connected directly to the center pin of the cavity at a node of the standing-wave voltage distribution of the fundamental mode. (d) Sub- $\mu$ m-size electron trap near voltage maximum of the fundamental mode with constriction of width $500 \mathrm{~nm}$. (e) Circuit schematic showing the voltage distribution of the fundamental mode (red) and the simplified measurement and control circuit connected to the center pin (pink). The cavity is measured in transmission using a low-noise amplifier (LNA) and the trap potential is tuned through a dc source connected to the center pin (pink) through a low-pass filter. (f) Cross-sectional view of the cavity waveguide gap showing the schematic trap geometry. The ground planes (gray) form a microchannel of height $d=800 \mathrm{~nm}$ and width $w_{G}=6 \mu \mathrm{m}$ filled with superfluid ${ }^{4} \mathrm{He}$ by capillary action. A dc voltage on the submerged center pin (pink) of width $w_{\mathrm{cp}}=2 \mu \mathrm{m}$ and thickness $t=80 \mathrm{~nm}$ creates a parabolic trapping potential for electrons above the surface that couple to the rf field in the cavity.

raises the effective dielectric constant of the waveguide, lowering the resonator frequency proportionally to the thickness $h$.

To measure the cavity response to superfluid helium, we monitor the resonance frequency and quality factor in transmission while increasing the bulk helium reservoir level in small increments (the center pin is held at ground potential throughout this measurement). The results of such a helium filling experiment are presented in Fig. 2(a). Four different regimes can be clearly distinguished in the frequency shift [see Fig. 2(b)]. For small amounts of superfluid (regime I), an unsaturated van der Waals film of thickness $h \sim 30 \mathrm{~nm}$ forms as the liquid evenly coats the 


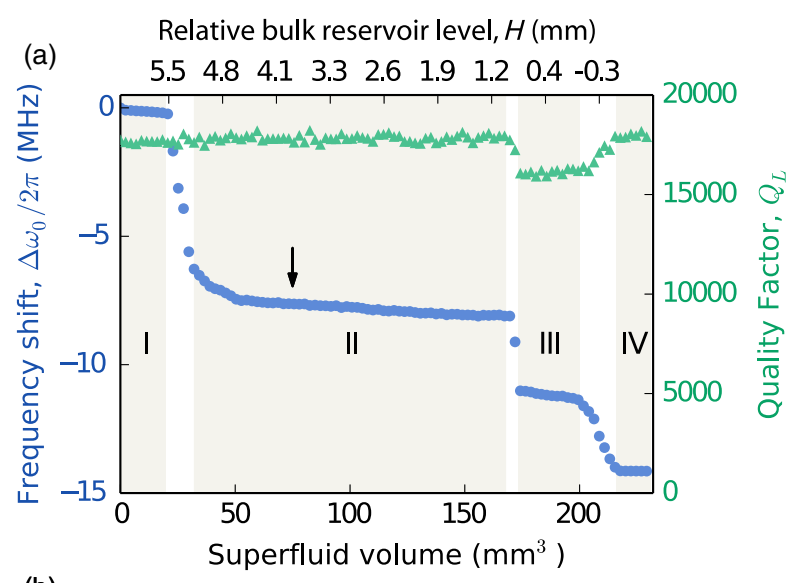

(b)

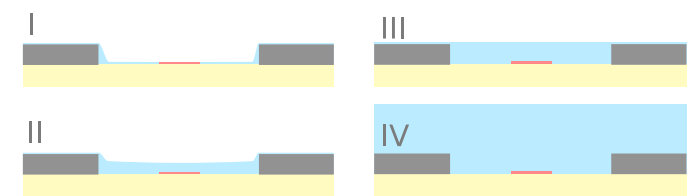

(c)

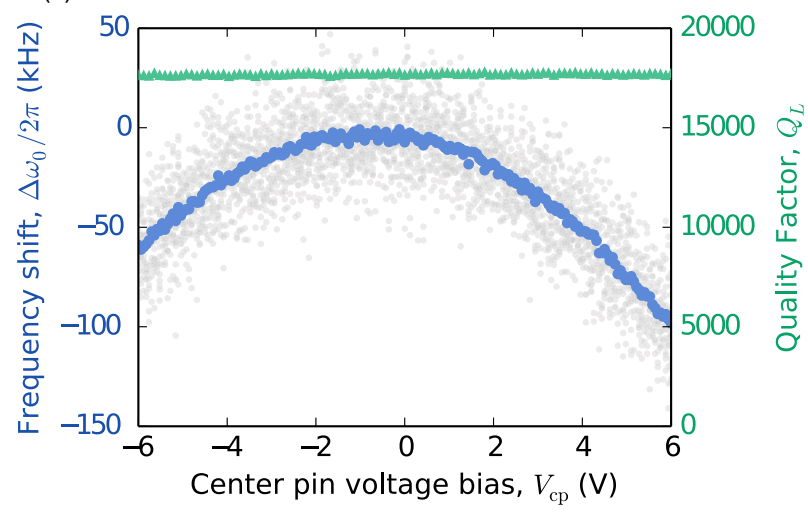

FIG. 2. Cavity response to superfluid helium. (a) Measured resonance frequency shifts $\Delta \omega_{0} / 2 \pi$ (blue dots, left axis) and loaded quality factor $Q_{L}$ (green triangles, right axis) as functions of superfluid volume supplied to the cell (bottom axis) and relative bulk helium level $H$ in the reservoir (top axis). Each data point corresponds to an increase in superfluid volume of $\Delta V_{\mathrm{sf}} \sim$ $2.3 \mathrm{~mm}^{3}$ and reservoir level $\Delta H \sim 70 \mu \mathrm{m}$. (b) Different filling state corresponding to the different regimes in (a). (c) Frequency shift (blue dots, left axis) and quality factor (green triangles, right axis) as functions of center pin voltage bias $V_{\mathrm{cp}}$ at fixed helium level in the capillary action regime indicated by arrow in (a). Gray dots are frequency shifts extracted from single-shot cavity transmission measurements with $N=80$ such measurements per voltage bias point $V_{\mathrm{cp}}$. The blue data points are averages over the single-shot measurements at each point.

surface of the resonator and the interior of the sample cell, leading to small frequency shifts of $\Delta \omega_{0}(h) / 2 \pi \simeq$ $-190 \mathrm{kHz}$. Once the van der Waals film has saturated, the liquid film shape is determined by capillary action with a semicircular profile $z(x) \sim x^{2} / 2 R_{c}(H)$ in the gap. The capillary radius $R_{c}(H)=\sigma / \rho g H$ is determined by the distance between the bulk helium level in the reservoir and the chip surface $H$, where $\sigma=0.378 \times 10^{-3} \mathrm{~N} / \mathrm{m}$ is the surface tension of liquid helium in vacuum, $\rho=$ $0.154 \times 10^{-3} \mathrm{~kg} / \mathrm{cm}^{3}$ the mass density, and $g$ is the gravitational acceleration. When the radius of curvature becomes on the order of the gap width $R_{c} \sim w_{G}$, the gap starts to fill up by capillary action and is filled completely for $R_{c} \gg w_{G}$ (regime II). Small increases in shift in the subsequent "flat" regime are due to decrease in the curvature of the helium profile. Finite element simulations show that when the channel is filled $(h=800 \mathrm{~nm})$ the frequency shift is $-8 \mathrm{MHz}$, in good agreement with the observed data. As $H \rightarrow 0$, the radius of curvature becomes on the order of the chip dimensions and eventually starts to diverge. We attribute the abrupt jump at $170 \mathrm{~mm}^{3}$ to the formation of a thick film that spans the entire chip (regime III) and is supported by the sample holder. As $R_{c} \rightarrow \infty$, the superfluid film becomes sensitive to mechanical vibrations and small fluctuations in the reservoir level, which manifests itself in a perceived drop in quality factor in this regime. Once the reservoir has been completely filled, the helium fills the region above the chip linearly (regime IV) until the resonator becomes insensitive at thickness $h \sim 6 \mu \mathrm{m}$, corresponding to a frequency shift of $\Delta \omega_{0}(h) /$ $2 \pi=-14.145 \mathrm{MHz}$, again in good agreement with numerical simulations that predict a final frequency shift of $-14.1 \mathrm{MHz}$. All subsequent experiments are done at the filling level indicated by the black arrow in Fig. 2(a), which corresponds to $h \approx 647 \mathrm{~nm}$, where the frequency shift is $\Delta \omega_{0}(h) / 2 \pi \simeq-7.4 \mathrm{MHz}$, and is only slightly changed by additional fluid introduced to the reservoir.

The superfluid level in the resonator gap can be modulated in situ by sweeping the voltage of the center pin $V_{\mathrm{cp}}$. To lowest order, the equilibrium film thickness at the center of the gap is determined by the electromechanical force on the film surface and surface tension with a quadratic voltage dependence $h\left(V_{\mathrm{cp}}\right) \approx h(0)+\left(V_{\mathrm{cp}}^{2} / 16 \sigma\right)$ $\left(\varepsilon_{\mathrm{He}}-\varepsilon_{0}\right)$. Figure 2(c) shows measured frequency shift and quality factor as functions of center pin voltage at a reservoir level of $H \simeq 4 \mathrm{~mm}$, where the gap is partly filled by capillary action. The resonance frequency shows a parabolic voltage dependence while the quality factor remains constant, as expected. The maximum observed shift at $V_{\mathrm{cp}}=+6 \mathrm{~V}$ of $\Delta \omega_{0} / 2 \pi=-100 \mathrm{kHz}$ corresponds to a change in film thickness of $\Delta h \simeq 13 \mathrm{~nm}$ at the center of the gap. The slight offset of the otherwise symmetric response is not well understood, and it is device dependent. The frequency sensitivity to level changes in the gap can be estimated from finite element electromagnetic simulations to be $\approx 8 \mathrm{kHz} / \mathrm{nm}$, consistent with the overall slope and frequency shift. The stability of the capillary action film is estimated from consecutive single-shot transmission measurements [gray data points in Fig. 2(c)], with $N=80$ frequency measurements per voltage bias point. Slow fluctuations of the helium level are manifested in the $\delta \omega_{0}^{(\mathrm{rms})} / 2 \pi=16 \mathrm{kHz}$ scatter of resonance frequencies 
[gray points in Fig. 2(c)] corresponding to helium level fluctuations of $\delta h^{(\mathrm{rms})} \approx 2.6 \mathrm{~nm}$. The demonstrated electrostatic control over the helium level can be used to dynamically reduce the fluctuations.

In summary, the microwave measurement provides a high bandwidth way to measure the helium level and its fluctuations, down to $\mathrm{pm} / \sqrt{\mathrm{Hz}}$-level sensitivity. Using this technique, we establish a lithographically defined, stabilized superfluid helium film within the cavity trap. In the regime of the experiment, the channel helium level is insensitive to the small differences in the amount of helium put into the sample box. The measured helium level fluctuations are relatively small and will be monitored to see if they have a significant effect on the electron coherence time through changes in the trapping potential.

\section{DISPERSIVE MEASUREMENTS OF ELECTRONS IN A CAVITY}

Following uncharged superfluid measurements, we proceed to load electrons into the cavity mode volume and detect the trapped electron ensemble in transmission measurements. Electrons are generated via pulsed thermionic emission from a tungsten filament mounted in vacuum above the device and attracted towards the superfluid surface in the resonator channel by a positive trap voltage $V_{\mathrm{cp}}$. After waiting for the sample to cool, the cavity transmission is monitored while tuning the center pin voltage $V_{\text {cp }}$ (Fig. 3) starting from $+3 \mathrm{~V}$. The dispersive interaction of the cavity with the trapped ensemble leads to a voltage-dependent shift of the cavity resonance towards lower frequencies before reverting back at negative trap potentials. We observe maximum resonance shifts of up to $\Delta \omega>10 \kappa$ cavity linewidths in frequency while $Q$ is somewhat reduced [Fig. 3(b)]. The electron-induced frequency shift reaches a maximum of $\Delta \omega_{\max } / 2 \pi=$ $-2.47 \mathrm{MHz}$ at $V_{\mathrm{cp}}^{(\text {th) }}=+0.91 \mathrm{~V}$ with a drop in quality factor and a corresponding increase in cavity decay rate of $\Delta \kappa_{\max } / 2 \pi=122 \mathrm{kHz}$ [blue curves in Figs. 3(c) and 3(d)]. These changes in the cavity resonance frequency are at least an order of magnitude larger than those caused by the electric-field-induced helium film thickness change without electrons. Below the threshold $V_{\mathrm{cp}}^{\text {(th) }}$, the electron-induced shifts decrease gradually as electrons are lost from the trapping region. To ensure that the observed cavity response is due to the trapped electron ensemble, we perform a control experiment where the filament is fired while the center pin is biased at $-1 \mathrm{~V}$. The voltage is then swept in the reverse direction [red curves in Figs. 3(c) and 3(d)]. The voltagedependent signal is completely absent [see Fig. 2(b) and discussion above]. The ensemble-induced cavity response has been reproduced in independent experiments using five different devices. The maximum observed resonance shifts are repeatable and generally vary between 2 and $8 \mathrm{MHz}$ based on loading conditions.
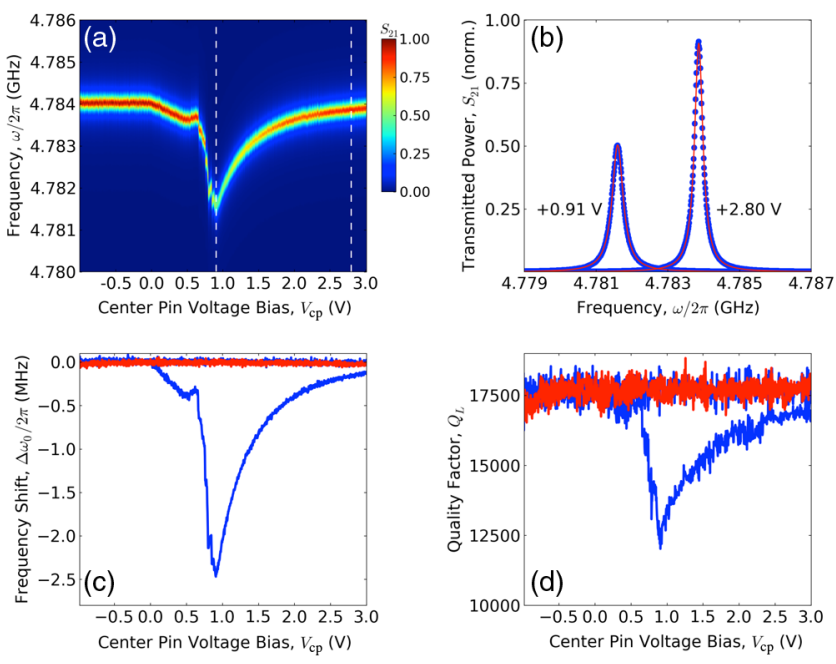

FIG. 3. Detection of a trapped electron ensemble on superfluid helium in a cavity transmission experiment. (a) Normalized transmitted power through the cavity as a function of center pin trap voltage $V_{\mathrm{cp}}$. (b) Normalized transmission spectra at $V_{\text {cp }}=+0.91$ and $+2.8 \mathrm{~V}$, showing a shift in resonance frequency and a reduction in transmitted power at the bias points indicated by the dashed vertical lines in (a). Solid red lines are fits to Lorentzians. (c) Resonance frequency and (d) loaded quality factor as functions of trap bias in the presence (blue) and absence (red) of an electron ensemble. In panels (a)-(d), electrons are first loaded into the cavity mode volume at an initial bias of $V_{\mathrm{cp}}=$ $+3 \mathrm{~V}$ and a fixed helium level in the capillary regime with an uncharged shift of $\Delta \omega_{0} / 2 \pi=-7.58 \mathrm{MHz}$ and a reservoir level of $H \simeq 4 \mathrm{~mm}$. The blue line shows the trap voltage being swept from +3 to $-1 \mathrm{~V}$ and back in $4 \mathrm{mV}$ steps, eventually depleting the trap region, while the red line shows the same sweep for an empty trap.

To further investigate the quantitative form of the cavity shift as a response to the number of electrons $N$ and the bias voltage $\mathrm{V}_{\mathrm{cp}}$, we design a protocol to partially drain the electrons from the trap. In the following experiment, at the end of the first sweep from $+3 \mathrm{~V}$ (bottom half of blue curve in Fig. 4), we deliberately sweep down below the $V_{\mathrm{cp}}^{(\text {th })}$ to introduce electron loss. Now, with fewer electrons in the trap (top half of blue curve in Fig. 4), the cavity resonance shift is smaller in magnitude than before, but nonhysteretic unless another electron-loss event is triggered. For subsequent sweeps, we then set successively lower stop voltages. The final sweep (orange curve in Fig. 4) depletes the mode volume of all electrons as evidenced by the vanishing frequency shift on the final upward sweep. By carefully tuning the stop voltages and number of sweeps we can controllably reduce the number of electrons as desired.

A quantitative understanding of the electron-cavity interaction requires modeling of the classical many-body interactions between electrons as well as the coupling of the resulting electron normal modes with the cavity. We develop a nonperturbative numerical model that determines 


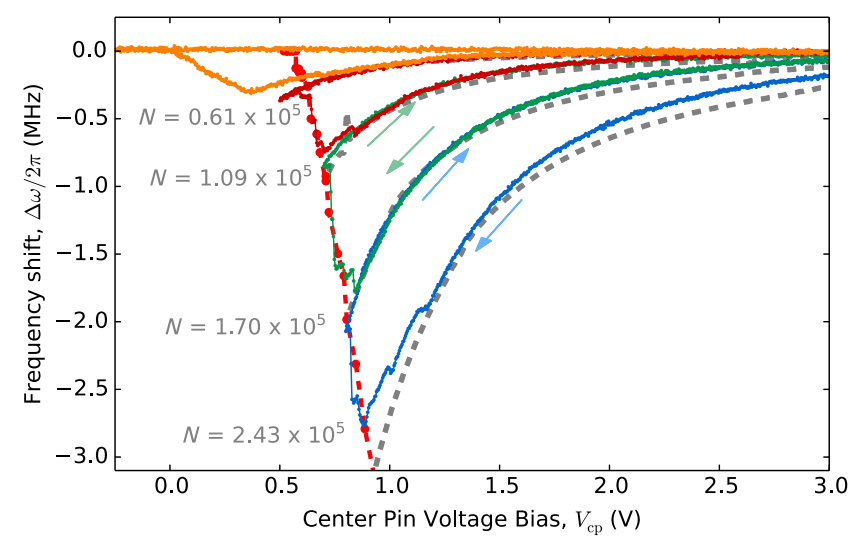

FIG. 4. Measured cavity frequency shifts as a function of trap voltage bias $V_{\mathrm{cp}}$. Colors indicate consecutive cycles of the voltage sweep. In each cycle $V_{\mathrm{cp}}$ is decreased (arrows) until electrons are irreversibly lost from the trap and then increased to the initial value of $3.0 \mathrm{~V}$. Gray dashed lines in the background show frequency shifts predicted by molecular dynamics simulation. For each of the four isoelectron number curves the number of electrons in the simulation is depicted to the left. A red dotted line indicates modeled electron loss with respect to a leak voltage of (530) $\mathrm{mV}$. The isoelectron number curves from the experiment terminate within a small neighborhood of this loss frontier.

the electron ensemble configuration, frequencies, and coupling to the cavity. First, simulated annealing is employed to find the minimal energy configuration of the electrons. The electrostatic potential used in this step is constructed using field profile data derived from the sample geometry. After obtaining the electron configurations, we solve the equations of motion of the cavity-electron coupled system in a nonperturbative way to obtain the cavity frequency shift. For a given number of electrons in the trap, this calculation is repeated for various $V_{\mathrm{cp}}$ to produce the corresponding frequency shift curve. Curves for various numbers of electrons are computed, and no fitting parameters are used in the model besides picking the closest isoelectron number curve. Figure 4 shows excellent agreement between the data and our computational model. The model is described in detail in the Supplemental Material [36].

Using the measured signal and our model, we can infer the electron mode frequency and coupling strength $g_{\mathrm{rms}}$. The frequency of the strongest coupled mode grows proportionally to $\sqrt{V_{\mathrm{cp}}}$ and is roughly $25 \mathrm{GHz}$ at $V_{\mathrm{cp}}=0.5 \mathrm{~V}$, which shows we are well within the dispersive regime. In this regime, the cavity frequency shift is related to the rms singleelectron coupling by $\Delta \omega=N g_{\mathrm{rms}}^{2} /\left(\omega_{e}-\omega_{0}\right)$, where $\omega_{e}$ is the electron motional frequency, $\omega_{0}$ is the cavity frequency, and $N$ is the number of electrons. We determine both $\omega_{e}$ and $N$ by comparing the data from Fig. 4 to simulation results (see Supplemental Material [36]) and find $g_{\mathrm{rms}} / 2 \pi=$ $0.8 \mathrm{MHz}$ for a $1-\mu \mathrm{m}$-wide electron configuration. In future trap designs, additional guard electrodes can improve trap stability at low voltage allowing access to the resonant regime. The coupling, though quite large already, can be enhanced further by shrinking the dimensions of the trap.

There are two types of electron loss observed. The first, which determines the number of electrons loaded, occurs at higher $V_{\mathrm{cp}}$ due to hydrodynamic instability [33]. The density immediately after loading at $V_{\mathrm{cp}}=3 \mathrm{~V}$ is $n \approx 2 \times 10^{9} \mathrm{~cm}^{-2}$. The second type of electron loss occurs when the trap depth becomes sufficiently shallow, such that electrons can leak out of the trap. We model this phenomenologically by assuming that electrons are lost if the potential difference between the ground plane and electrons is less than $V_{\text {leak }}$. Using $V_{\text {leak }}$ as a single-fit parameter in the molecular dynamics simulation, we find best agreement between simulation and experiment when $V_{\text {leak }}=530 \mathrm{mV}$. The residual population for small electron numbers at small $V_{\text {cp }}$ (orange curve in Fig. 4) is not well understood.

In summary, we demonstrate the successful trapping and detection of an electron ensemble above the surface of superfluid helium in the circuit QED architecture. The measurement technique we introduce here could extend traditional electrons on helium experiments to smaller ensembles and enable observation of the electron dynamics. The observation of the large dispersive shift and the good agreement with our numerical simulations indicate that it should be possible to perform cavity QED experiments in a single electron quantum dot. Though small, the fluctuations in the helium film thickness are an important source of decoherence for the electron motional states, and merit further study. Finally, the sensitivity of the device to helium thickness changes can be exploited for novel cavity optomechanics experiments with superfluid ripplons.

\section{ACKNOWLEDGMENTS}

The authors thank Mark Dykman, Steve Lyon, and David G. Rees for many helpful discussions, and the HOOMD molecular dynamics simulation package [37-39]. The authors thank C. Suzanne Miller and Ralu Divan for support with device fabrication. This work was supported by NSF CAREER Grant No. DMR 1151839, the University of Chicago MRSEC program of the NSF under Award No. DMR 1420709, and the David and Lucile Packard Foundation. Use of the Center for Nanoscale Materials, an Office of Science user facility, was supported by the U.S. Department of Energy, Office of Science, Office of Basic Energy Sciences, under Contract No. DEAC02-06CH11357.

[1] S. A. Lyon, Spin-Based Quantum Computing Using Electrons on Liquid Helium, Phys. Rev. A 74, 052338 (2006).

[2] P. M. Platzman and M. I. Dykman, Quantum Computing with Electrons Floating on Liquid Helium, Science 284, 1967 (1999). 
[3] M. I. Dykman, P. M. Platzman, and P. Seddighrad, Qubits with Electrons on Liquid Helium, Phys. Rev. B 67, 155402 (2003).

[4] D. I. Schuster, A. Fragner, M. I. Dykman, S. A. Lyon, and R. J. Schoelkopf, Proposal for Manipulating and Detecting Spin and Orbital States of Trapped Electrons on Helium Using Cavity Quantum Electrodynamics, Phys. Rev. Lett. 105, 040503 (2010).

[5] Y. Monarkha and K. Kono, Two-Dimensional Coulomb Liquids and Solids (Springer, Berlin, 2004).

[6] K. Shirahama and K. Kono, Dynamical Transition in the Wigner Solid on a Liquid Helium Surface, Phys. Rev. Lett. 74, 781 (1995).

[7] K. Shirahama, S. Ito, H. Suto, and K. Kono, Surface Study of Liquid ${ }^{3}$ He Using Surface State Electrons, J. Low Temp. Phys. 101, 439 (1995).

[8] R. Williams, R.S. Crandall, and A. H. Willis, Surface States of Electrons on Liquid Helium, Phys. Rev. Lett. 26, 7 (1971).

[9] C. C. Grimes and G. Adams, Evidence for a Liquid-toCrystal Phase Transition in a Classical, Two-Dimensional Sheet of Electrons, Phys. Rev. Lett. 42, 795 (1979).

[10] E. Y. Andrei, Two-Dimensional Electron Systems (Springer, Netherland, 1997), Vol. 19.

[11] H. Ikegami, Y. Tsutsumi, and K. Kono, Chiral Symmetry Breaking in Superfluid ${ }^{3} \mathrm{He}-A$, Science 341, 59 (2013).

[12] K. Kono, Electrons on the Surface of Superfluid ${ }^{3}$ He, J. Low Temp. Phys. 158, 288 (2010).

[13] A. D. Chepelianskii, M. Watanabe, K. Nasyedkin, K. Kono, and D. Konstantinov, An Incompressible State of a Photoexcited Electron Gas, Nat. Commun. 6, 7210 (2015).

[14] G. Papageorgiou, P. Glasson, K. Harrabi, V. Antonov, E. Collin, P. Fozooni, P. G. Frayne, M. J. Lea, D. G. Rees, and Y. Mukharsky, Counting Individual Trapped Electrons on Liquid Helium, Appl. Phys. Lett. 86, 153106 (2005).

[15] E. Rousseau, D. Ponarin, L. Hristakos, O. Avenel, E. Varoquaux, and Y. Mukharsky, Addition Spectra of Wigner Islands of Electrons on Superfluid Helium, Phys. Rev. B 79, 045406 (2009).

[16] F. R. Bradbury, M. Takita, T. M. Gurrieri, K. J. Wilkel, K. Eng, M.S. Carroll, and S. A. Lyon, Efficient Clocked Electron Transfer on Superfluid Helium, Phys. Rev. Lett. 107, 266803 (2011).

[17] M. Takita and S. A. Lyon, Isolating Electrons on Superfluid Helium, J. Phys. Conf. Ser. 568, 052034 (2014).

[18] L. P. Gor'kov and D. M. Chernikova, Concerning the Structure of a Charged Surface of Liquid Helium, JETP Lett. 18, 119 (1973).

[19] V. S. Edel'man, Levitated Electrons, Sov. Phys. Usp. 23, 227 (1980).

[20] J. R. Petta, A. C. Johnson, J. M. Taylor, E. A. Laird, A. Yacoby, M. D. Lukin, C. M. Marcus, M. P. Hanson, and A. C. Gossard, Coherent Manipulation of Coupled Electron Spins in Semiconductor Quantum Dots, Science 309, 2180 (2005).

[21] A. Wallraff, D. I. Schuster, A. Blais, L. Frunzio, R. S. Huang, J. Majer, S. Kumar, S. M. Girvin, and R. J. Schoelkopf, Strong Coupling of a Single Photon to a Superconducting Qubit Using Circuit Quantum Electrodynamics, Nature (London) 431, 162 (2004).
[22] A. Blais, R.-S. Huang, A. Wallraff, S. M. Girvin, and R. J. Schoelkopf, Cavity Quantum Electrodynamics for Superconducting Electrical Circuits: An Architecture for Quantum Computation, Phys. Rev. A 69, 062320 (2004).

[23] W. Sommer and D. Tanner, Mobility of Electrons on the Surface of Liquid ${ }^{4} \mathrm{He}$, Phys. Rev. Lett. 27, 1345 (1971).

[24] R. J. Schoelkopf and S. M. Girvin, Wiring Up Quantum Systems, Nature (London) 451, 664 (2008).

[25] M. H. Devoret and R. J. Schoelkopf, Superconducting Circuits for Quantum Information: An Outlook, Science 339, 1169 (2013).

[26] Y. Kubo, F. R. Ong, P. Bertet, D. Vion, V. Jacques, D. Zheng, A. Dréau, J.-F. Roch, A. Auffeves, F. Jelezko, J. Wrachtrup, M. F. Barthe, P. Bergonzo, and D. Esteve, Strong Coupling of a Spin Ensemble to a Superconducting Resonator, Phys. Rev. Lett. 105, 140502 (2010).

[27] D. I. Schuster, A. P. Sears, E. Ginossar, L. DiCarlo, L. Frunzio, J. J. L. Morton, H. Wu, G. A. D. Briggs, B. B. Buckley, D. D. Awschalom, and R. J. Schoelkopf, HighCooperativity Coupling of Electron-Spin Ensembles to Superconducting Cavities, Phys. Rev. Lett. 105, 140501 (2010).

[28] S. Probst, H. Rotzinger, S. Wünsch, P. Jung, M. Jerger, M. Siegel, A. V. Ustinov, and P. A. Bushev, Anisotropic Rare-Earth Spin Ensemble Strongly Coupled to a Superconducting Resonator, Phys. Rev. Lett. 110, 157001 (2013).

[29] H. Malissa, D. I. Schuster, A. M. Tyryshkin, A. A. Houck, and S. A. Lyon, Superconducting Coplanar Waveguide Resonators for Low Temperature Pulsed Electron Spin Resonance Spectroscopy, Rev. Sci. Instrum. 84, 025116 (2013).

[30] K. D. Petersson, L. W. McFaul, M. D. Schroer, M. Jung, J. M. Taylor, A. A. Houck, and J. R. Petta, Circuit Quantum Electrodynamics with a Spin Qubit, Nature (London) 490, 380 (2012).

[31] T. Frey, P. J. Leek, M. Beck, A. Blais, T. Ihn, K. Ensslin, and A. Wallraff, Dipole Coupling of a Double Quantum Dot to a Microwave Resonator, Phys. Rev. Lett. 108, 046807 (2012).

[32] J. J. Viennot, M. C. Dartiailh, A. Cottet, and T. Kontos, Coherent Coupling of a Single Spin to Microwave Cavity Photons, Science 349, 408 (2015).

[33] D. Marty, Stability of Two-Dimensional Electrons on a Fractionated Helium Surface, J. Phys. C 19, 6097 (1986).

[34] D. G. Rees, I. Kuroda, C. A. Marrache-Kikuchi, M. Höfer, P. Leiderer, and K. Kono, Point-Contact Transport Properties of Strongly Correlated Electrons on Liquid Helium, Phys. Rev. Lett. 106, 026803 (2011).

[35] A. D. Armour, M. P. Blencowe, E. Brahimi, and A. J. Rimberg, Universal Quantum Fluctuations of a Cavity Mode Driven by a Josephson Junction, Phys. Rev. Lett. 111, 247001 (2013).

[36] See Supplemental Material at http://link.aps.org/ supplemental/10.1103/PhysRevX.6.011031 for details of the classical many-body model of electrons in a channel coupled to a CPW. 
[37] J. A. Anderson, C. D. Lorenz, and A. Travesset, General Purpose Molecular Dynamics Simulations Fully Implemented on Graphics Processing Units, J. Comput. Phys. 227, 5342 (2008).

[38] HOOMD, http://codeblue.umich.edu/hoomd-blue.
[39] J. Glaser, T. D. Nguyen, J. A. Anderson, P. Liu, F. Spiga, J. A. Millan, D. C. Morse, and S. C. Glotzer, Strong Scaling of General-Purpose Molecular Dynamics Simulations on GPUs, Comput. Phys. Commun. 192, 97 (2015). 